\title{
Cross-sectional imaging of pendeo-epitaxial GaN using continuous-wave two-photon microphotoluminescence
}

\author{
P. J. Schuck ${ }^{\mathrm{a})}$ and R. D. Grober \\ Yale University, Applied Physics, Becton Center, New Haven, Connecticut 06520
}

\author{
A. M. Roskowski, S. Einfeldt, and R. F. Davis \\ North Carolina State University, Raleigh, North Carolina 27695
}

(Received 10 May 2002; accepted for publication 18 July 2002)

\begin{abstract}
A technique utilizing continuous-wave two-photon absorption has been developed for optically sectioning and imaging deep into $\mathrm{GaN}$ structures. Imaging at depths greater than $20 \mu \mathrm{m}$ below the surface of a coalesced pendeo-epitaxial GaN sample is demonstrated. Free and donor-bound excitonic emission in this sample appears to originate at the surface, acceptor-bound exciton transitions are strongest in the top bulk portion of the sample, and subgap luminescence is most intense deep in the sample. The depth resolution of the imaging system is measured to be $1.75 \mu \mathrm{m}$ near the GaN surface. (C) 2002 American Institute of Physics. [DOI: 10.1063/1.1506948]
\end{abstract}

The optical properties of the III-nitride material system have garnered increased attention in recent years due to the promise of the III-nitrides for short wavelength optoelectronic device applications. ${ }^{1}$ As a tool for optical characterization, photoluminescence (PL) has proved invaluable in studying such disparate material properties as band structure, impurity identification and concentration, and material quality. One drawback of traditional PL experiments on $\mathrm{GaN}$, however, is that the UV radiation used for excitation is absorbed in the top 50-100 nm of the material. ${ }^{2,3}$ This means that only surface and near-surface properties are examined, leaving the bulk properties to be studied by more indirect methods. Motivated by this limitation, we have developed a technique, based on the two-photon absorption (TPA) process, which enables the observation of PL at any depth inside a $\mathrm{GaN}$ structure.

In this letter, single-photon PL will refer to PL experiments where the absorption of a single photon excites an electron into the conduction band. For this case, the energy of the pump photon is greater than the band gap, $E_{g}$, of the sample. Two-photon PL, on the other hand, will describe PL that is initiated by a TPA event. In this case, the energy from two photons is required to excite a carrier into the conduction band. The energy of the pump photons will be between $E_{g} / 2$ and $E_{g}$, assuming a single-excitation source.

The operating principle behind our technique, and a primary advantage of TPA experiments, is that the pump radiation can penetrate to any depth within the sample since the energy of the pump photons is smaller than the GaN band gap. Significant absorption occurs only within the sample volume at the focal point of the focusing optic where the pump intensity is greatest. ${ }^{4}$ By scanning the focal volume relative to the sample, it is possible to examine optical properties and material quality at the surface, in the bulk, and at interfaces deep within $\mathrm{GaN}$ structures. The dimensions of the absorption volume are defined by both the response function of the optical system and the intensity dependence of the absorption coefficient. For TPA, probability for absorption is proportional to intensity squared. If one assumes a linear

${ }^{a)}$ Electronic mail: james.schuck@yale.edu relationship between absorption and luminescence, then twophoton PL emission should also be proportional to the square of the pump intensity.

Our data is acquired using a home-built scanning confocal UV microscope. The samples sit at a low temperature $(\sim 10 \mathrm{~K})$ inside a liquid helium, continuous-flow Janis microscope cryostat. The $325 \mathrm{~nm}$ line of a $\mathrm{He}-\mathrm{Cd}$ laser is used for single-photon excitation while the 488 line from an argon-ion laser is used for two-photon excitation. We both excite and collect through a $0.6 \mathrm{NA}, 40 \times$ Zeiss microscope objective that corrects for the spherical aberration encountered when passing through a sapphire window above the sample. Collected emission is focused through a pinhole, defining our spatial resolution, and dispersed inside one of two grating monochromators. The spectra are detected with a liquid-nitrogen-cooled charge-coupled device (CCD) camera. In this work, the $X-Y$ plane is defined to be parallel to the sample surface.

TPA experiments are generally performed using pulsed excitation sources (e.g., femtosecond lasers) in order to achieve the high instantaneous power densities that are required. In our experiments, however, we observe two-photon PL from GaN using a continuous wave (cw) argon-ion laser as the excitation source. There are two main reasons for this. The first is that when compared to other wide band gap semiconductors, ${ }^{5} \mathrm{GaN}$ has a relatively large two-photon cross section, $\beta$, of approximately $15 \mathrm{~cm} / \mathrm{GW} .{ }^{6-10}$ Second, because our microscope is designed to achieve high spatial resolution, we can focus our excitation to a small, diffraction limited spot, thus providing the considerable power densities that are needed for two-photon experiments. The use of a cw laser source also simplifies our system as no femtosecond laser oscillators are required.

Figure 1 is a typical GaN spectrum we detect using our $\frac{1}{4}$ meter Acton monochromator when we excite with the 488 $\mathrm{nm}$ laser line. The laser line can be seen toward the righthand side of the spectrum, where it has saturated the CCD camera. To the right-hand side of the laser line, $\mathrm{GaN}$ and $\mathrm{SiC}$ Raman lines are present, as well as yellow-band PL. Note, however, that emission is also detected to the left-hand side of the laser line. This emission is at energies higher than that 


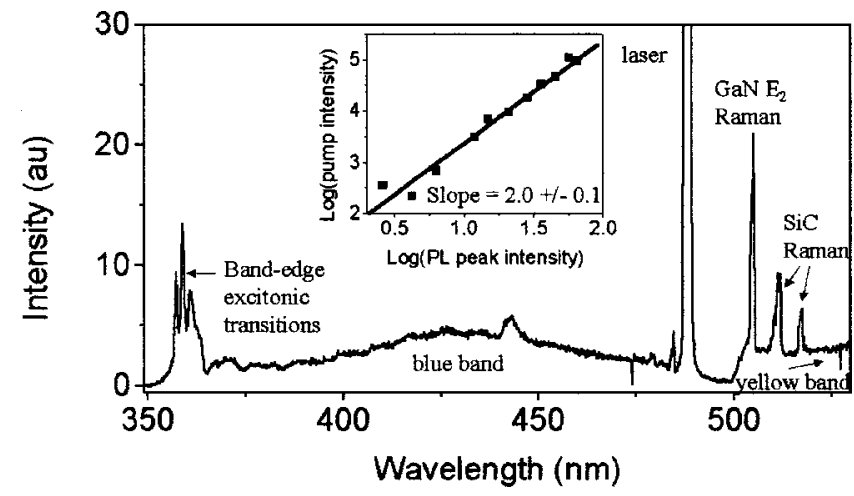

FIG. 1. PL and Raman spectrum of GaN at $T=10 \mathrm{~K}$ when excited with the $488 \mathrm{~nm}$ argon-ion laser line. The luminescence that occurs at wavelengths shorter that $488 \mathrm{~nm}$ is a result of TPA. The peak band-edge PL intensity dependence on pump intensity is shown as a $\log -\log$ plot in the inset. The slope of the fitted line equals 2 , confirming the occurrence of TPA.

of the excitation source, and is due to TPA. Both band-edge excitonic transitions and blue-band luminescence are observed. The inset in Fig. 1 is a $\log -\log$ plot of pump intensity versus band-edge luminescence peak intensity. The slope of $2.0 \pm 0.1$ of the fitted line confirms that TPA is the cause of the high-energy emission. Figure 1 illustrates the potential power of the two-photon excitation technique; it is possible to investigate band-edge, blue, and yellow luminescence as well as Raman scattering, simultaneously.

To characterize our system, we chose to study a coalesced maskless pendeo-epitaxial GaN sample. Details regarding pendeo-epitaxial GaN growth can be found in Refs. 11 and 12. A cross-sectional scanning electron microscopy (SEM) image of our sample is shown in Fig. 2. This sample was chosen for a variety of reasons: First, pendeo-epitaxial growth has been shown to result in material with a dislocation density less than $10^{5} \mathrm{~cm}^{-2}$. Second, at $23 \mu \mathrm{m}$, the sample is much thicker than the depth resolution of our system, allowing us to quantify the imaging capabilities of this technique. Third, the sample has physical structure that changes as a function of depth. In particular, there are periodic voids in the material that begin at a depth of roughly 10 $\mu \mathrm{m}$ and widen as they get closer to the substrate.

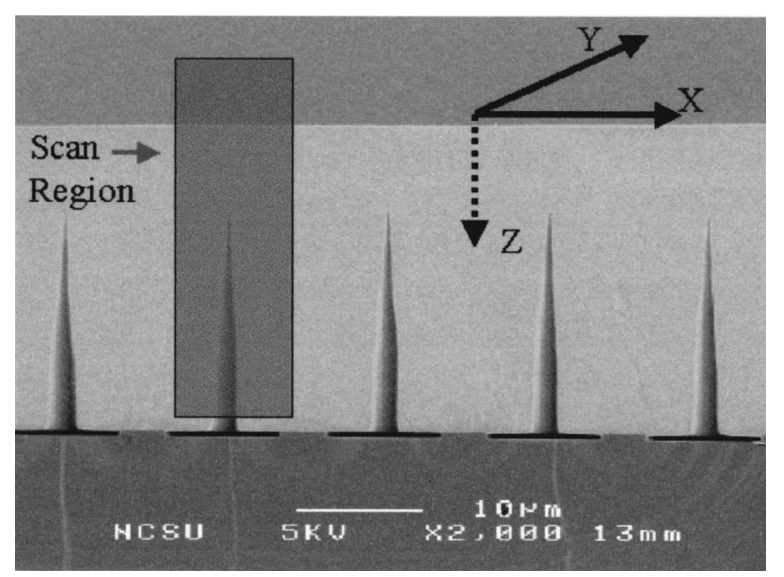

FIG. 2. Cross-sectional SEM of the coalesced pendeo-epitaxial GaN sample. Here, the $Z$ axis is the axis perpendicular to the substrate. Note the presence of periodic voids in the material that begin at a depth of approximately $10 \mu \mathrm{m}$. The two-photon depth scans encompass regions similar to

the one specified by the shaded box.

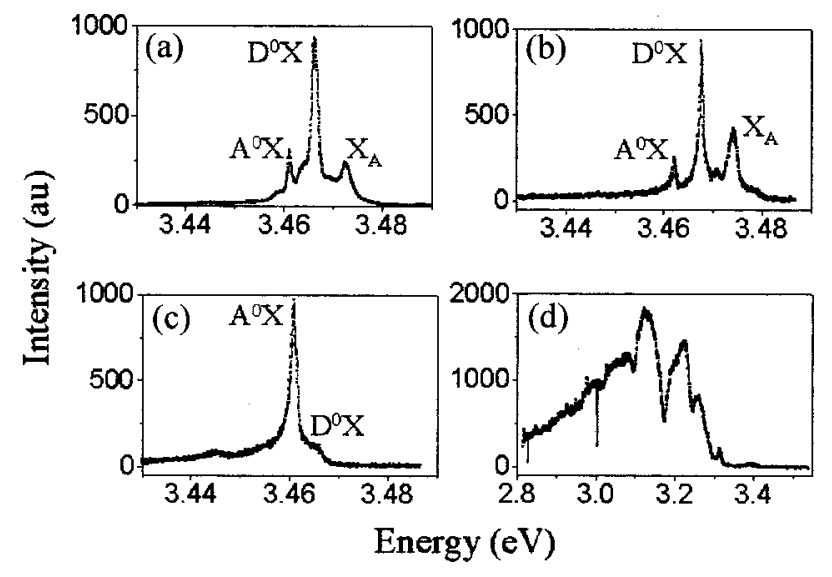

FIG. 3. Spectra acquired from different depths within the sample. Both (a) and (b) are surface spectra, where the $325 \mathrm{~nm}$ line from a $\mathrm{He}-\mathrm{Cd}$ laser is used as the pump in (a) and the $488 \mathrm{~nm}$ argon-ion line is used as the pump in (b). Spectra (c) and (d) (488 nm pump for both) originate at depths of 5 $\mu \mathrm{m}$ and $20 \mu \mathrm{m}$, respectively.

As alluded to previously, traditional single-photon micro-PL $(\mu$-PL) data is accumulated from $X-Y$ scans of the sample surface due to absorption. By employing two-photon excitation, we perform $X-Z$ scans of our sample; that is, we scan into the material creating cross-sectional images from the collected luminescence. Our typical scan covers an area similar to that specified by the shaded region in Fig. 2. The data collection procedure and analysis for these types of scans is described in greater detail in Ref. 13.

Figures 3(a)-3(d) are select spectra taken from different depths within the sample. The band-edge spectra in Figs. 3(a)-3(c) were collected using a 2400 line/mm grating inside a $1 \mathrm{~m}$ Spex monochromator. Figure 3(a) is a single-photon PL spectrum. Figures 3(b) and 3(c) are two-photon PL spectra, with Fig. 3(b) acquired at the surface of the sample and Fig. 3(c) acquired at a depth of approximately $5 \mu \mathrm{m}$ below the surface. The peaks in Figs. 3(a) and 3(b) at 3.474, 3.468, and $3.462 \mathrm{eV}$ correspond to the free $A$ exciton $\left(X_{A}\right)$, donorbound exciton $\left(D^{0} X\right)$, and acceptor-bound exciton $\left(A^{0} X\right)$, respectively, with the $D^{0} X$ peak being the strongest. Other spectral features are present, but will not be discussed here. In Fig. 3(c) (depth of $5 \mu \mathrm{m}$ ), the $A^{0} X$ transition dominates and is stronger than it is in Fig. 3(b) (at the surface). The $D^{0} X$ transition in Fig. 3(c) is now only a shoulder to the right-hand side of the $A^{0} X$ peak, and the $X_{A}$ peak has disappeared. The similarity of spectra in Figs. 3(a) and 3(b) and the dissimilarity between Figs. 3(a) and 3(c) demonstrates that single-photon PL experiments investigate only nearsurface material properties. To investigate midgap emission over a wide spectral range, a 600 lines $/ \mathrm{mm}$ grating inside a $1 / 4$ meter monochromator was used. Figure 3(d) is from a depth of roughly $20 \mu \mathrm{m}$ and shows strong luminescence in the range between 2.8 and $3.3 \mathrm{eV}$.

The images in Figs. 4(a)-4(e) are from a single $X-Z$ two-photon $\mu$-PL scan of the sample. Each is an image of integrated PL intensity from an energy window centered at the specified energy and approximately $0.6 \mathrm{meV}$ wide. These images demonstrate that different emission energies originate from different depths within the sample. Figures 4(a) and 4(b) are images of PL from 3.474 and $3.468 \mathrm{eV}$, and are associated with $X_{A}$ and $D^{0} X$ emission, respectively. EmisAIP license or copyright; see http://apl.aip.org/apl/copyright.jsp 


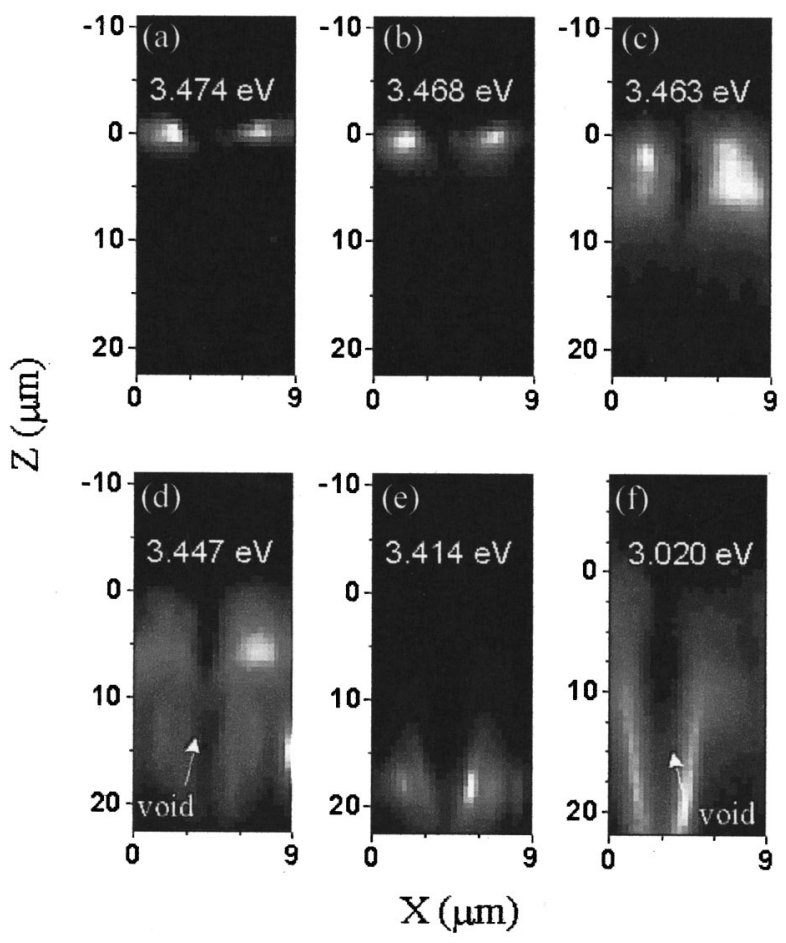

FIG. 4. Images of PL intensity centered at the indicated energies. The images in (a), (b), and (c) are associated with $X_{A}, D^{0} X$, and $A^{0} X$ transitions, respectively.

sion at these energies appears only near the surface. An intensity linecut of $X_{A}$ emission in the $Z$ direction in Fig. 4(a) gives an upper limit to the depth resolution of our system of $1.75 \mu \mathrm{m}$ (full width at half maximum). It should be noted that the depth resolution in this system is diffraction limited only near the top of the sample. Deep in the sample, refraction effects will dominate ${ }^{13}$ due to the index mismatch between air and $\mathrm{GaN}(n \approx 2.55$ at $488 \mathrm{~nm}){ }^{14}$

Figure 4(c) is an image of $A^{0} X$ luminescence. Unlike $X_{A}$ and $D^{0} X$ emission, $A^{0} X$ transitions appear to originate in the top bulk portion of the sample. In Fig. 4(d), we observe $3.447 \mathrm{eV}$ luminescence from areas throughout most of the sample. Notice that the void is clearly visible in Fig. 4(d). At lower energies, emission comes from even deeper in the sample. PL centered at $3.414 \mathrm{eV}$ is localized at the sample bottom, near the sample-substrate interface, as seen in Fig. 4(e).

The data for Figs. 4(a)-4(e) were acquired with a $1 \mathrm{~m}$ monochromator so as to obtain high spectral resolution. All energies associated with these images, therefore, are from the band-edge and near-band-edge region of the spectrum. The data for Fig. 4(f) were collected using the 1/4 meter monochromator so as to investigate the broad midgap emission we observe deep in the sample. As can be seen in Fig. 4(f), midgap luminescence that is centered around $3 \mathrm{eV}$ originates mostly from the bottom half of the sample and is strongest in the wing regions near the void.

We would like to address some possible explanations for the emission behavior observed in Fig. 4. As is clearly seen in Fig. 4, depth of emission correlates with photon energy. Chromatic effects in our optics would result in different focal depths in the sample depending on the energy of the emitted light and could, therefore, be a potential explanation. While chromatic aberration is a serious issue in any two-photon experiment, however, it is unlikely to account for our data due to the relatively small wavelength range (roughly $7 \mathrm{~nm}$ ) that is investigated in Figs. 4(a)-4(e).

The increase in midgap emission intensity at greater depths does correlate with increased dislocation density. ${ }^{15,16}$ While material quality may account for the lower-energy luminescence behavior, it is less likely that it is related to the surface emission behavior of the $X_{A}$ and $D^{0} X$. A possible explanation for the observed surface luminescence may be optical reabsorption; band-edge transitions may only be detected near the surface because high-energy photons emitted in the bulk are absorbed before they leave the sample. This explanation is supported by absorption data in literature where absorption depths, even at energies $10 \mathrm{meV}$ lower than the $X_{A}$ absorption peak, are less than $0.25 \mu \mathrm{m} .{ }^{3}$ Another possible explanation, however, could be that this sample contains a high density of donors at the surface. At this point, though, the exact origin of the observed emission behavior is unresolved.

In conclusion, we have demonstrated a cw two-photon imaging technique by generating cross-sectional PL images of a $23 \mu \mathrm{m}$ thick pendeo-epitaxial $\mathrm{GaN}$ sample. We observe free and donor-bound excitonic emission only at the surface, while subgap luminescence is strongest deep in the sample. Whether this observed emission behavior is due to optical reabsorption, a high density of donors near the surface, or a combination of both factors is still an open question. We have measured the depth resolution of our system to be less than $1.75 \mu \mathrm{m}$, though increasing the NA of the imaging optics will result in significant improvement of the depth resolution.

This work is supported by the Office of Naval Research MURI on Polarization Electronics Contract No. N00014-961-1179, under the direction of Dr. Colin E. C. Wood.

${ }^{1}$ S. Nakamura, M. Senoh, S. Nagahama, and N. Iwasa, Jpn. J. Appl. Phys. Part 1 34, 799 (1995).

${ }^{2}$ R. Dingle, D. D. Sell, S. E. Stokowski, and M. Ilegems, Phys. Rev. B 4, 1211 (1971)

J. F. Muth, J. H. Lee, I. K. Shmagin, R. M. Kolbas, H. C. Casey, Jr., B. P. Keller, U. K. Mishra, and S. P. DenBaars, Appl. Phys. Lett. 71, 2572 (1997)

${ }^{4}$ W. Denk, J. H. Strickler, and W. W. Webb, Science 248, 73 (1990).

${ }^{5}$ E. W. Van Stryland, M. A. Woodall, H. Vanherzeele, and M. J. Soileau, Opt. Lett. 10, 490 (1985); and references cited therein.

${ }^{6}$ J. Miragliota and D. K. Wickenden, Appl. Phys. Lett. 69, 2095 (1996).

${ }^{7}$ A. M. Streltsov, K. D. Moll, A. L. Gaeta, P. Kung, D. Walker, and M. Razeghi, Appl. Phys. Lett. 75, 3778 (1999).

${ }^{8}$ C. K. Sun, J. C. Liang, J. C. Wang, F. J. Kao, S. Keller, M. P. Mack, U. Mishra, and S. P. DenBaars, Appl. Phys. Lett. 76, 439 (2000).

${ }^{9}$ Y. L. Huang, C. K. Sun, J. C. Liang, S. Keller, M. P. Mack, U. K. Mishra, and S. P. Denbaars, Appl. Phys. Lett. 75, 3524 (1999).

${ }^{10}$ V. Pacebutas, A. Stalnionis, A. Krotkus, T. Suski, P. Perlin, and M. Leszczynski, Appl. Phys. Lett. 78, 4118 (2001).

${ }^{11}$ T. Zheleva, S. Smith, D. Thomson, K. Linthicum, P. Rajagopal, and R. F. Davis, J. Electron. Mater. 28, L5 (1999).

${ }^{12}$ A. M. Roskowski, E. A. Preble, S. Einfeldt, P. M. Miraglia, and R. F. Davis, J. Electron. Mater. 31, 421 (2002).

${ }^{13}$ P. J. Schuck, M. D. Mason, R. D. Grober, O. Ambacher, A. P. Lima, C. Miskys, R. Dimitrov, and M. Stutzmann, Appl. Phys. Lett. 79, 952 (2001).

${ }^{14}$ N. J. Everall, Appl. Spectrosc. 54, 773 (2000).

${ }^{15}$ M. E. Lin, B. N. Sverdlov, S. Strite, H. Mokoc, and A. E. Drakin, Electron. Lett. 29, 1759 (1993).

${ }^{16}$ Z. Liliental-Weber (personal communication). 